\title{
MINIMAL COHESIVE BASIC SETS
}

\author{
by DONALD L. GOLDSMITH $\dagger$
}

(Received 21st March 1973)

\section{Introduction}

A basic set (formerly basic sequence) $\mathscr{B}$ is a set of pairs $(a, b)$ of positive integers satisfying

(1) if $(a, b) \in \mathscr{B}$, then $(b, a) \in \mathscr{B}$,

(2) $(a, b c) \in \mathscr{B}$ if and only if $(a, b) \in \mathscr{B}$ and $(a, c) \in \mathscr{B}$,

(3) $(1, k) \in \mathscr{B}, k=1,2, \ldots$

Some familiar examples of basic sets are

$\mathscr{S}=\bigcup_{k=1}^{\infty} S_{k}$, where $S_{k}=\{(1, k),(k, 1)\}$,

$\mathscr{U}=\{(a, b) \mid a$ and $b$ are relatively prime positive integers $\}$,

$\mathscr{L}=\{(a, b) \mid a$ and $b$ are any positive integers $\}$.

If $\Phi$ is any set of pairs of positive integers, the basic set generated by $\Phi$ is the intersection of all basic sets which contain $\Phi$. If $\mathscr{B}$ is generated by $\Phi$, we write

$$
\mathscr{B}=\Gamma[\Phi] .
$$

A pair $(p, q)$ is called a primitive pair if both $p$ and $q$ are primes.

A basic set $\mathscr{B}$ is cohesive if, for each positive integer $k$, there is an integer $a=a(k)>1$ such that $(k, a) \in \mathscr{B}$. $\mathscr{M}$ and $\mathscr{L}$ are cohesive, as is the basic set

$$
\mathscr{B}\left[p^{*}\right]=\Gamma\left[\bigcup_{q \in P}\left(p^{*}, q\right)\right]
$$

generated by the primitive pairs $\bigcup_{q \in P}\left(p^{*}, q\right)$, where $p^{*}$ is any fixed prime and $P$ is the set of all primes. $\mathscr{B}$ is minimally cohesive provided

(1) $\mathscr{B}$ is cohesive,

(2) if $\mathscr{B}^{\prime} \subset \mathscr{B}$ but $\mathscr{B}^{\prime} \neq \mathscr{B}$ then $\mathscr{B}^{\prime}$ is not cohesive.

The function-theoretic and combinatorial properties of arbitrary basic sets were discussed in (1) and (2), and those of cohesive basic sets in (3). We confine ourselves in this note to a further investigation of the combinatorial

$\dagger$ This research was partially supported by a Western Michigan University Faculty Research Fellowship. 
properties of cohesive basic sets. In particular, we give a complete determination of the collection of all minimal cohesive basic sets and show that there are no minimal cohesive basic sets contained in $\mathscr{M}$.

Our principal result is the following

Theorem. $A$ basic set $\mathscr{B}$ is minimally cohesive if and only if $\mathscr{B}=\mathscr{B}\left[p^{\star}\right]$ for some prime $p^{*}$.

\section{Proof of the Theorem}

We will use several lemmas leading to our main result.

Lemma 1. Suppose that $\mathscr{B}$ is cohesive and that $\Phi_{\mathscr{B}}$ is the set of all primitive pairs in $\mathscr{B}$. Then $\mathscr{B}$ is minimally cohesive if and only if

$$
\mathscr{B}(p, q)=\Gamma\left[\Phi_{\mathscr{B}}-\{(p, q),(q, p)\}\right]
$$

is not cohesive for every primitive pair $(p, q)$ in $\mathscr{B}$.

Lemma 2. If $\mathscr{B}$ is cohesive and $\mathscr{B} \subset \mathscr{M}$, then for every positive integer $k$ there are infinitely many primes $r$ such that $(k, r) \in \mathscr{B}$.

Lemma 3. There are no minimal cohesive basic sets in $\mathscr{M}$.

Proof. Let $\mathscr{B}$ be any cohesive basic set contained in $\mathscr{M}$, and let $\left(p_{0}, q_{0}\right)$ be any primitive pair in $\mathscr{B}$. Choose any integer $k>1$. By Lemma 2 , there is a prime $r$ different from $p_{0}$ and $q_{0}$ for which $(k, r) \in \mathscr{B}$. For each prime divisor $p$ of $k,(p, r) \in \mathscr{B}$ and also $(p, r) \in \mathscr{B}\left(p_{0}, q_{0}\right)$. Hence $(k, r) \in \mathscr{B}\left(p_{0}, q_{0}\right)$. It follows that $\mathscr{B}\left(p_{0}, q_{0}\right)$ is cohesive and so, by Lemma $1, \mathscr{B}$ is not minimally cohesive. That proves Lemma 3.

For a basic set $\mathscr{B}$ and a positive integer $k$, let $C_{\mathscr{B}}(k)$ denote the set of prime companions of $k$ in $\mathscr{B}$; that is,

$$
C_{\mathscr{B}}(k)=\{p \mid p \in P,(p, k) \in \mathscr{B}\} .
$$

Note that $\mathscr{B}$ is cohesive if and only if $C_{\mathscr{B}}(k)$ is never empty for any $k$.

We are now ready for the proof of the main theorem.

Suppose $p^{*}$ is a fixed (but arbitrary) prime and let $\mathscr{B}=\mathscr{B}\left[p^{*}\right]$. $\mathscr{B}$ is clearly cohesive since $\left(k, p^{*}\right) \in \mathscr{B}$ for every positive integer $k$. Moreover, $\mathscr{B}\left(p^{*}, q\right)$ is not cohesive for any prime $q$. For if $q \neq p^{*}$, then $C_{\mathscr{B}\left(p^{*}, q\right)}(q)$ is empty, and if $q=p^{*}$, then $C_{\mathscr{B}\left(p^{*}, p^{*}\right)}\left(p^{*} q^{\prime}\right)$ is empty, where $q^{\prime}$ is any prime different from $p^{*}$. Therefore $\mathscr{B}\left[p^{*}\right]$ is minimally cohesive.

Conversely, suppose that $\mathscr{B}$ is any minimally cohesive basic set. It is sufficient to show that the primitive pairs $\left(p^{*}, q\right)$ are in $\mathscr{B}$ for some fixed prime $p^{*}$ and every prime $q$ in $P$.

By Lemma $3, \mathscr{B} \not \subset \mathscr{M}$. Set

$$
\mathscr{B}_{1}=\mathscr{B} \cap \mathscr{M} .
$$

$\mathscr{B}_{1}$ is a basic subset of $\mathscr{M}$, and we assert that $\mathscr{B}_{1}$ is not cohesive. For if $(p, q)$ is any primitive pair in $\mathscr{B}_{1}$, then also $(p, q) \in \mathscr{B}$, and Lemma 1 and the minimal 
cohesiveness of $\mathscr{B}$ imply that $\mathscr{B}(p, q)$ is not cohesive. But $\mathscr{B}_{1}(p, q) \subset \mathscr{B}(p, q)$, and so $\mathscr{B}_{1}(p, q)$ is not cohesive. Therefore if $\mathscr{B}_{1}$ were cohesive, then by Lemma 1 it would be a minimally cohesive basic subset of $\mathscr{M}$, contrary to Lemma 3 .

Since $\mathscr{B}_{1}$ is not cohesive, there is an integer $k_{0}>1$ such that, for every integer $a>1,\left(k_{0}, a\right) \notin \mathscr{B}_{1}$. Now if $p$ is any prime in $C_{\mathscr{B}}\left(k_{0}\right)$, then $p$ must divide $k_{0}$, for otherwise $p$ and $k_{0}$ would be relatively prime, so $\left(p, k_{0}\right) \in \mathscr{B} \cap \mathscr{M}=\mathscr{B}_{1}$, contrary to the choice of $k_{0}$. In particular $C_{\mathscr{g}}\left(k_{0}\right)$ is finite.

Enumerate all the primes: $q_{1}, q_{2}, \ldots$, and set

$$
R_{l}=C_{\mathscr{B}}\left(k_{0}\right) \cap C_{\mathscr{B}}\left(q_{1}\right) \cap C_{\mathscr{B}}\left(q_{2}\right) \cap \ldots \cap C_{\mathscr{B}}\left(q_{l}\right),
$$

for $l=1,2, \ldots$ We assert that $R_{l} \neq \varnothing(l=1,2, \ldots)$. Since $\mathscr{B}$ is cohesive, there is an integer $a>1$ for which

$$
\left(a, k_{0} q_{1} q_{2} \ldots q_{l}\right) \in \mathscr{B} \text {. }
$$

It follows that $\left(a, k_{0}\right) \in \mathscr{B}$, so each prime divisor of $a$ is in $C_{\mathscr{A}}\left(k_{0}\right)$. Moreover, $\left(a, q_{i}\right) \in \mathscr{B}(i=1, \ldots, l)$, so each prime divisor of $a$ is also in $C_{\mathscr{B}}\left(q_{i}\right)(i=1, \ldots, l)$. Hence $R_{l} \neq \varnothing$, as claimed.

It follows from the preceding that $\left\{R_{l}\right\}_{l=1}^{\infty}$ is a nested, decreasing sequence of non-empty, finite sets of primes. Therefore

$$
R=\bigcap_{l=1}^{\infty} R_{l} \neq \varnothing .
$$

If $p^{*}$ is any prime in $R$, then $\left(p^{*}, q\right) \in \mathscr{B}$ for every prime $q$ in $P$.

That completes the proof of the theorem.

\section{REFERENCES}

(1) D. L. GoldsMith, On the multiplicative properties of arithmetic functions, Pacific J. Math. 27 (1968), 283-304.

(2) D. L. GolDSMITH, On the structure of certain basic sequences associated with an arithmetic function, Proc. Edinburgh Math. Soc. 17 (1971), 305-310.

(3) A. A. GiolA and D. L. Goldsmith, Convolutions of arithmetic functions over cohesive basic sequences, Pacific J. Math. 38 (1971), 391-399.

Western Michigan UNIVERSITY

Kalamazoo, Michigan 49001 